\title{
Uptake of a mixture of amino acids by mouse blastocysts
}

\author{
V. K. Lamb and H. J. Leese \\ Department of Biology, University of York, Heslington, York, YO1 5DD, UK
}

\begin{abstract}
Preimplantation mouse embryos exhibit growth at the blastocyst stage when there is a dramatic increase in protein content between days 4 and 5 after hCG treatment. This increase in protein synthesis requires the uptake of amino acids from the surrounding medium, but the consumption of individual amino acids present as a mixture has not been examined. We therefore measured the simultaneous depletion of 18 amino acids by mouse blastocysts in culture on days 4 and 5 after hCG treatment. Two culture media were used: one with amino acids present at concentrations largely based on those reported to give optimal rates of embryo development (M16/mix AA), and a second with the amino acids each at $0.1 \mathrm{mmol}^{-1}$ (M16/0.1 AA). After derivatization with o-phthaldialdehyde to yield highly fluorescent products, amino acid concentrations in the media were measured by HPLC. On day 4, seven amino acids were depleted from M16/mix AA at rates significantly greater than zero: aspartate, arginine, glycine, alanine, isoleucine, leucine and lysine; on day 5, nine amino acids were depleted at significant rates: aspartate, glutamate, asparagine, tyrosine, methionine, valine, phenylalanine, isoleucine and leucine. Average amino acid depletion from MI6/mix AA was $32 \%$ greater on day 5 than on day 4. Aspartate, glutamate, arginine, isoleucine and leucine appeared to be the key amino acids sought by embryos on both days tested. Embryos grown in amino-acid-containing media appeared morphologically normal and their volume increased compared with embryos cultured in the absence of amino acids.
\end{abstract}

\section{Introduction}

Exogenously supplied amino acids have been shown to affect the development of preimplantation mammalian embryos in vitro (Spindle and Pedersen, 1973; Carney and Bavister, 1987; Gardner and Lane, 1993), and have been detected in the female reproductive tract (Leese et al., 1979; Miller and Schultz, 1987). The uptake and incorporation of amino acids by embryos increases during preimplantation development (Brinster, 1971; Leese, 1991). For example, the $V_{\max }$ of transport system $b^{0,+}$, a sodium-independent carrier for cationic and neutral amino acids, increases by a factor of 30 between the one-cell and blastocyst stages (Van Winkle et al., 1990a). Moreover, changes in most of the amino acid transport systems occur at the blastocyst stage, and there is an accompanying increase in competition between amino acids for uptake (Van Winkle, 1988). The kinetics of amino acid transport into early embryos have been widely examined, notably in the mouse, for example, glycine transport (Hobbs and Kaye, 1985) and anionic amino acid transport (Van Winkle et al., 1991). The topic has been reviewed by Kaye (1986) and Van Winkle (1988).

Previous studies have relied on administering single, radiolabelled amino acids and there have been no reports on the uptake of a mixture of amino acids by early mouse embryos. We therefore incubated groups of mouse embryos with a mixture of 20 amino acids. As there are no reports on the complete amino acid composition of mouse uterine fluid, the environment in which blastocysts would normally be found, the concentrations of amino acids were largely based on those found to give optimal growth of mouse blastocysts (Spindle and Pedersen, 1973). In a second group of experiments, the amino acids were each present at $0.1 \mathrm{mmol} \mathrm{I}^{-1}$. Depletion rates for 18 of the amino acids have been followed on days 4 and 5 after hCG treatment and individual amino acids detected fluorometrically following HPLC.

Mouse blastocysts normally increase in volume from day 4 to day 5, and we therefore use blastocyst expansion as a quantitative criterion to determine whether exposure to the amino acid mixtures sustains embryo development.

\section{Materials and Methods}

\section{Embryos}

Embryos were obtained from 4-6-week-old CBA/Ca $\times \mathrm{C} 57 \mathrm{BL} / 6$ female mice mated with males of the same strain. Superovulation was induced by the injection i.p. of 5 iu pregnant mares' serum gonadotrophin (PMSG) (Intervet UK Ltd, Cambridge, UK), at the mid-point of the $12 \mathrm{~h}$ light period, followed $48 \mathrm{~h}$ later by 5 iu hCG (Intervet UK Ltd). The presence of a vaginal plug on day 1 indicated that mating had occurred. Embryos were collected on the morning of day 4 by flushing the uteri with medium M2 plus polyvinyl alcohol (PVA). Abnormal embryos were discarded. 
Table 1. Amino acid concentrations in the culture medium M16/mix AA for mouse blastocysts

\begin{tabular}{ll}
\hline Amino acid & Concentration $\left(\mathrm{mmol} \mathrm{l}^{-1}\right)$ \\
\hline Ala & \\
Arg & 0.56 \\
Asn & 0.10 \\
Asp & 0.15 \\
Cys & 0.45 \\
Gln & 0.23 \\
Glu & 0.50 \\
Gly & 0.88 \\
His & 1.00 \\
Ile & 0.50 \\
Leu & 0.20 \\
Lys & 1.00 \\
Met & 1.00 \\
Phe & 0.25 \\
Pro & 0.50 \\
Ser & 0.35 \\
Thr & 0.48 \\
Trp & 2.00 \\
Tyr & 0.10 \\
Val & 0.10 \\
& 1.00 \\
\hline
\end{tabular}

\section{Culture media}

The media were based on M2 (Quinn et al., 1982) and M16 (Whittingham, 1971), both supplemented with $1 \mathrm{~g} \mathrm{PVA} \mathrm{I}^{-1}$ (Sigma Chemical Co., Poole) in place of BSA. In medium M16/mix AA, amino acids (Sigma Chemical Co.) were present at the concentrations shown in Table 1 . The concentrations of essential amino acids were based on those found to be optimal for the development of blastocysts by Spindle and Pedersen (1973), and the nonessential amino acids on a combination of those found in medium 199 and the data of Miller and Schultz (1987) on the amino acid composition of rabbit oviduct and uterine fluids. In M16/0.1 AA, amino acids were each present at $0.1 \mathrm{mmol} \mathrm{l}^{-1}$.

The study was confined to those amino acids present in proteins; thus, compounds such as ornithine and taurine were not examined.

\section{Embryo culture}

Drops ( $1 \mu \mathrm{l}$ ) of M16, M16/mix AA and M16/0.1 AA under silicone fluid were pre-equilibrated under a humidified atmosphere of $5 \% \mathrm{CO}_{2}: 95 \%$ air at $37^{\circ} \mathrm{C}$. Embryos were washed three times in $\mathrm{M} 2$ and divided into three groups according to the medium in which they to be cultured: M16, M16/mix AA or M16/0.1 AA. The average number of embryos per drop on day 4 was 46,42 and 32 for M16, M16/mix AA and M16/0.1 AA, respectively. The embryos were washed three times in culture medium, taken up in $1 \mu \mathrm{l}$ of the same medium and added to a further $1 \mu$ l culture drop. One microlitre of medium was then removed so that the embryos were contained in a $1 \mu \mathrm{l}$ drop. This procedure was repeated with a second drop without embryos, which served as a control. Embryos were cultured under a humidified atmosphere containing $5 \% \mathrm{CO}_{2}: 95 \%$ air at $37^{\circ} \mathrm{C}$ for $4 \mathrm{~h}$. After incubation they were transferred to a fresh $1 \mu \mathrm{l}$ drop of medium and the above procedure repeated on the following day, when the number of embryos per $1 \mu \mathrm{l}$ drop was, on average, four fewer than that on day 4. After the incubations, the embryos were discarded.

The groups cultured in M16 served as controls for (1) the presence of contaminating amino acids in MI6 and (2) the appearance of embryo-derived amino acids in the culture drop. The data for amino acid depletion were corrected for these two criteria. Such corrections accounted for less than $5 \%$ of the total values.

\section{Analysis of amino acid content}

At the end of the incubation period, $0.5 \mu \mathrm{l}$ of medium was removed from each drop, diluted 1:25 with deionized water and frozen at $-20^{\circ} \mathrm{C}$ until analysed.

The amino acid analysis method was based on that of Jones et al. (1981) in which amino acids are reacted with $o$-phthaldialdehyde to yield highly fluorescent products, but has been greatly modified. The amino acids were analysed by reverse phase HPLC using two Waters pumps (model 510), a Beckman C18-ultrasphere ODS column, a Rheodyne 7125 injector with a $20 \mu \mathrm{l}$ injection loop, a Waters automated gradient controller, a Beckman 157 fluorescence detector and a Spectra-Physics SP 4270 integrator.

Reagent 1 consisted of $10 \mu \mathrm{l}$ 2-mercaptoethanol $(\mathrm{BDH}$, Poole) and $5 \mathrm{ml}$ o-phthaldialdehyde reagent solution (Sigma Chemical Co.). Reagent 2 was a solution of $0.1 \mathrm{~mol}$ potassium dihydrogen orthophosphate $\left.\left(\mathrm{KH}_{2} \mathrm{PO}_{4}\right)\right]^{-1}(\mathrm{BDH})$ adjusted to $\mathrm{pH}$ 4. These two reagents were stored at $-20^{\circ} \mathrm{C}$.

Reagent $1(10 \mu \mathrm{l})$ was added to $10 \mu \mathrm{l}$ of sample, left at room temperature for $1 \mathrm{~min}$ and the reaction stopped by adding $20 \mu$ l Reagent 2. The sample was immediately injected on to the column. The elution gradient, operated at a flow rate of $0.8 \mathrm{ml} \mathrm{min}^{-1}$, is shown in Fig. 1a. Solvent A consisted of $200 \mathrm{ml}$ methanol (Aldrich, Gillingham), $800 \mathrm{ml}$ sodium acetate $\left(83 \mathrm{mmol} \mathrm{l} \mathrm{l}^{-1}, \mathrm{pH} 5.9\right)(\mathrm{BDH})$ and $18 \mathrm{ml}$ tetrahydrafuran (Aldrich). Solvent B was $800 \mathrm{ml}$ methanol and $200 \mathrm{ml}$ sodium acetate ( $83 \mathrm{mmol} \mathrm{l}^{-1}, \mathrm{pH} \mathrm{5.9)}$.

The reference amino acid mixture was AA-S-18 (Sigma Chemical Co.) supplemented with tryptophan, asparagine and glutamine (Sigma Chemical Co.). All amino acids in the reference mixture were at a concentration of $12.5 \mu \mathrm{mol} \mathrm{I}^{-1}$. The elution profile (Fig. 1b) exhibited negligible day-to-day variation. An elution profile from a sample is shown in Fig. Ic. To enable accurate resolution of peaks, including complexes such as the triplet of histidine, serine and glutamine, the integrator assigned a code to each peak describing its characteristics. Peak areas were then calculated in a pre-determined manner according to the code assigned.

The peak areas for each amino acid were halved when the concentration of the reference mixture was halved; the response was thus taken to be linear. Amino acid concentrations were calculated by comparing peak areas in the standard with those in the sample.

With this technique, increases and decreases of less than $0.15 \mathrm{pmol}$ could be detected for each amino acid. Cysteine and proline could not be detected by this method. 

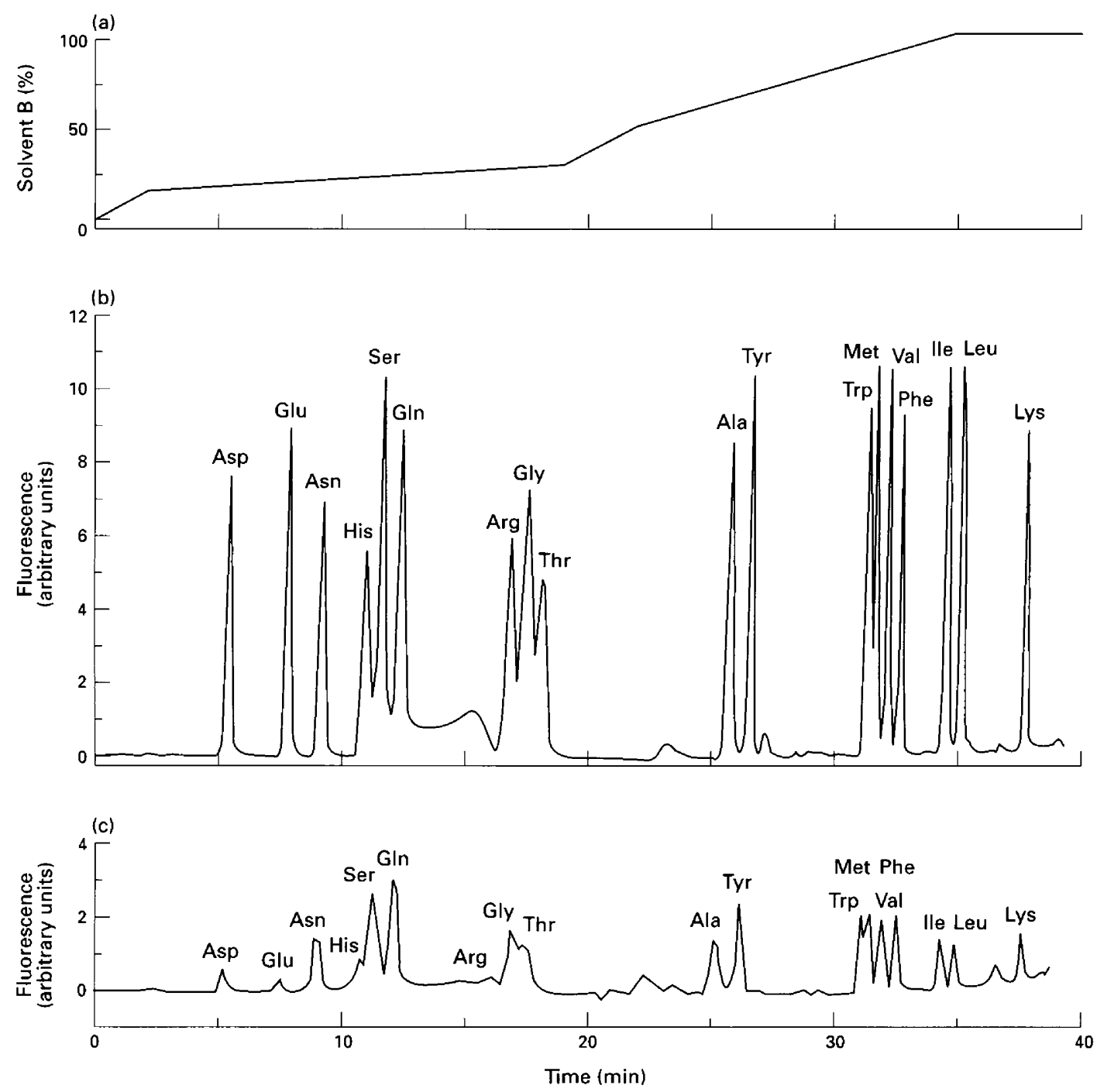

Fig. 1. (a) Elution gradient for amino acid separation. Solvent A: $200 \mathrm{ml}$ methanol, $800 \mathrm{ml}$ sodium acetate $\left(83 \mathrm{mmol} \mathrm{l}^{-1}, \mathrm{pH} \mathrm{5.9}\right), 18 \mathrm{ml}$ tetrahydrafuran. Solvent B: $800 \mathrm{ml}$ methanol, $200 \mathrm{ml}$ sodium acetate $\left(83 \mathrm{mmol} \mathrm{l}^{-1}\right.$, $\mathrm{pH}$ 5.9). Gradient programme: the programme was initiated with $0 \% \mathrm{~B}$, followed by a linear step to $15 \% \mathrm{~B}$ in $2 \mathrm{~min}$, a linear step to $28 \%$ B in $17 \mathrm{~min}$, a linear step to $50 \% \mathrm{~B}$ in $3 \mathrm{~min}$, and a linear step to $100 \% \mathrm{~B}$ in $13 \mathrm{~min}$. The flow rate was $0.8 \mathrm{ml} \mathrm{min}^{-1}$. (b) HPLC elution profile of a standard amino acid mixture. All amino acids were at $12.5 \mu \mathrm{mol} \mathrm{l}^{-1}$. (c) HPLC elution profile of M16/0.1 AA in which embryos had been cultured for $4 \mathrm{~h}$ on day 4 . All amino acids were at $0.1 \mathrm{mmol} \mathrm{l}^{-1}$ before incubation. Samples were derivatized with $o$-phthaldialdehyde before separation.

\section{Measurement of embryo volume}

On each day, the volume of embryos grown in M16 and M16/mix AA was calculated. A microscope eyepiece micrometer was calibrated against a haemocytometer grid. The long and short diameters of the embryos were measured. These values could then be substituted into the formula for the volume of a prolate spheroid: $V={ }_{3}^{4} \pi a b^{2}$; where $a=\frac{1}{2}$ long diameter and $b=\frac{1}{2}$ short diameter.

\section{Expression of results}

Results for M16/mix AA are expressed as absolute depletion rates of individual amino acids, in pmol per embryo $h^{-1}$, and also as the proportion of amino acids depleted, as percentage depletion per embryo $h^{-1}$, to take account of the different initial concentrations. Proportional depletion is calculated by dividing the absolute depletion rate by the initial concentration in the culture medium. Results for experiments involving M16/0.1 AA are expressed as proportional depletion rates only, since all the amino acids were present at the same initial concentration.

\section{Statistical analysis}

Values for depletion rates are means \pm SEM for five or six observations. Each depletion rate and proportional depletion 


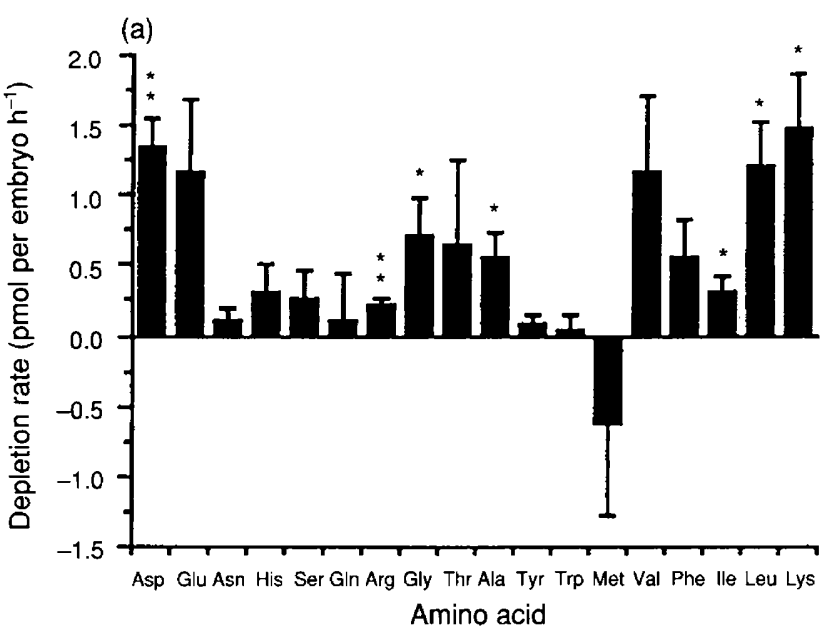

(b)

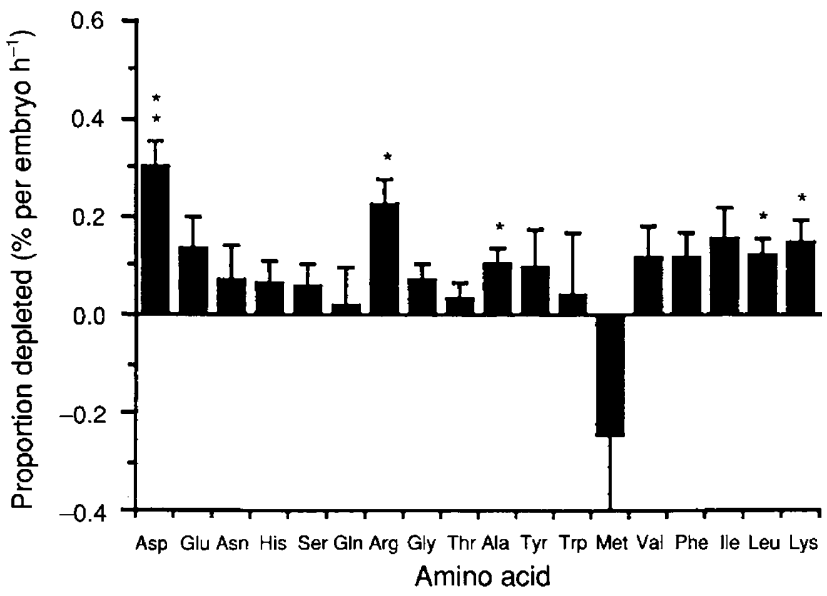

Fig. 2. Amino acid depletion rates by mouse embryos on day 4 incubated in the culture medium M16/mix AA for $4 \mathrm{~h}$. (a) Mean absolute amino acid depletion rates (pmol per embryo $h^{-1} \pm$ SEM). (b) Mean proportional amino acid depletion rates (\% per embryo $\mathrm{h}^{-1} \pm$ SEM. ${ }^{*} \mathrm{P}<0.05,{ }^{* *} \mathrm{P}<0.01$ compared with zero depletion.

rate was tested for significance from zero by Student's $t$ test. Values presented for embryo volumes are means \pm SEM for 10-15 observations. These data were analysed by Student's $t$ test, comparisons being made between media on each day.

\section{Results}

Day 4: depletion of amino acids from M16/mix AA

Glycine, alanine, isoleucine, leucine and lysine disappeared from the M16/mix AA medium at significant rates on day 4 $(P<0.05)$, while aspartate and arginine disappeared at highly significant rates $(P<0.01)$ (Fig. 2a). None of the other amino acids disappeared at a rate significantly different from zero. When considered as proportional depletion (Fig. 2b), only aspartate $(P<0.01)$, arginine, alanine, leucine and lysine $(P<0.05)$ were depleted from the medium at significant rates.

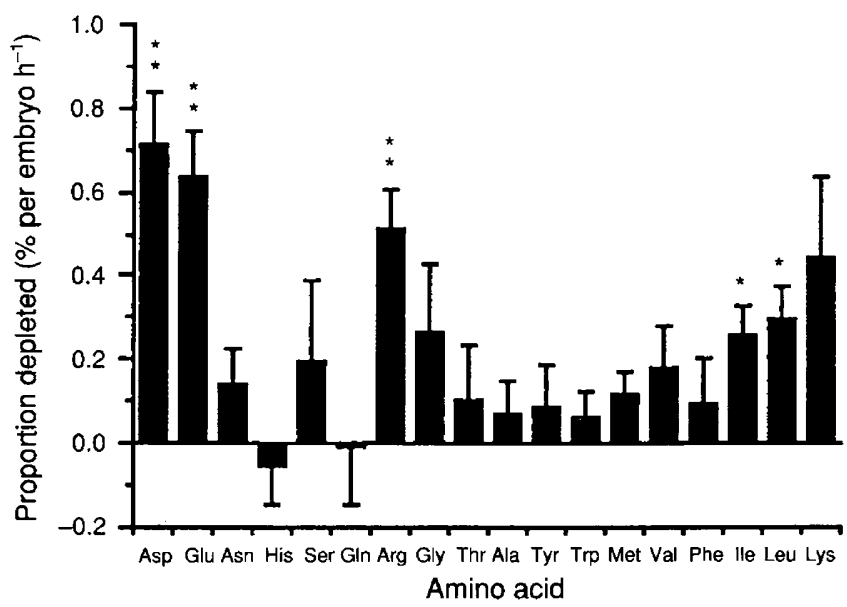

Fig. 3. Mean proportional amino acid depletion rates (\% per embryo $\mathrm{h}^{-1} \pm$ SEM) by mouse embryos on day 4 incubated in culture medium M16/0.1 AA for $4 \mathrm{~h} .{ }^{*} P<0.05,{ }^{* *} P<0.01$ compared with zero depletion.

Day 4: depletion from a mixture of amino acids all at $0.1 \mathrm{mmoll}^{-1}$ (M16/0.1 AA)

Five amino acids at $0.1 \mathrm{mmol} 1^{-1}$ were depleted from the M16/0.1 AA medium at significant rates: aspartate, glutamate and arginine $(P<0.01)$, isoleucine and leucine $(P<0.05)$ (Fig. 3).

\section{Day 5: depletion of amino acids from M16/mix $A A$}

Half of the amino acids from M16/mix AA were depleted on day 5 at rates significantly different from zero (Fig. 4a). Asparagine was significantly depleted $(P<0.05)$, and aspartate, glutamate, isoleucine and leucine were depleted at highly significant rates $(P<0.01)$, while tyrosine, methionine, valine and phenylalanine were depleted from the medium at very highly significant rates $(P<0.001)$. When the initial concentrations of amino acids in the medium were accounted for, the pattern of significance was very similar, with only glutamate and phenylalanine exhibiting a slightly less significant proportional depletion compared with absolute depletion (Fig. 4 b).

Day 5: depletion from a mixture of amino acids all at $0.1 \mathrm{mmoll}^{-1}$ (M16/0.1 AA)

Nine amino acids at $0.1 \mathrm{mmol} \mathrm{l}^{-1}$, aspartate, glutamate, threonine, tryptophan, methionine, valine, phenylalanine, isoleucine and leucine, were depleted from the M16/0.1 AA medium on day 5 at significant rates $(P<0.05)$, with a further two, asparagine and arginine, being depleted at highly significant rates $(P<0.01)$ (Fig. 5).

On both days, aspartate, isoleucine and leucine were depleted at significant absolute rates from M16/mix AA. Average absolute depletion from M16/mix AA was 32\% greater on day 5 than on day 4 . When considered as proportional depletion, aspartate and leucine were depleted at significant rates from both media on each day. On day 5, many more amino acids were depleted from both media than on day 4 . 


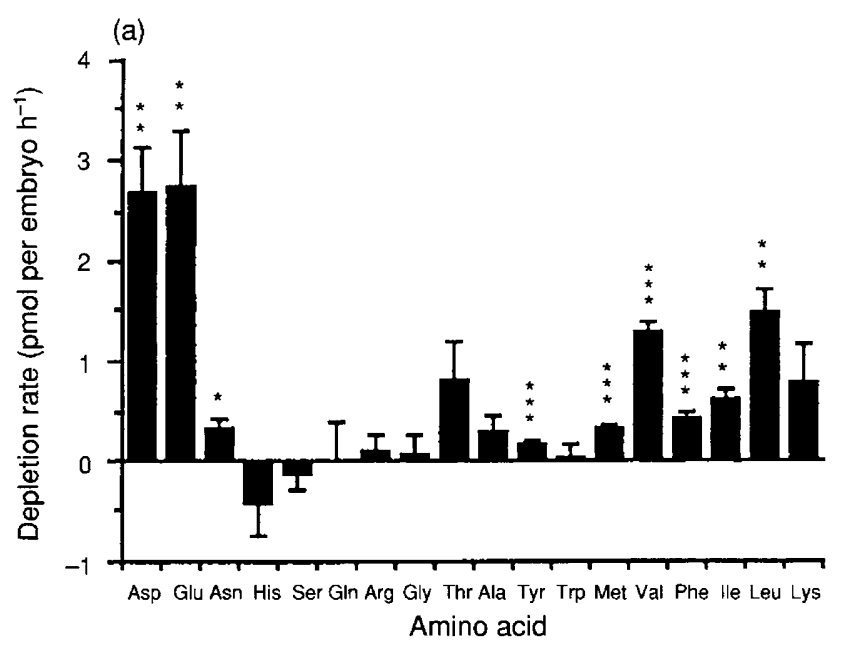

(b)

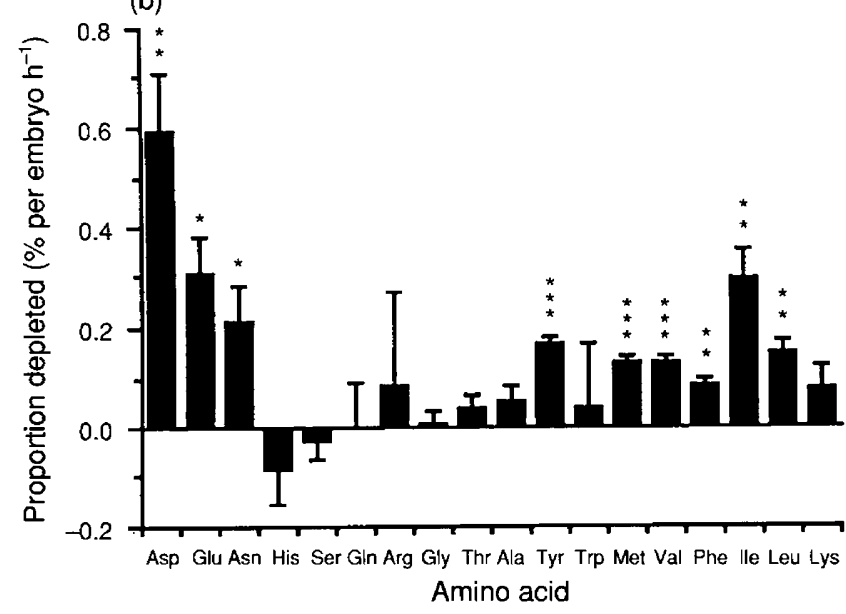

Fig. 4. Amino acid depletion rates by mouse embryos on day 5 incubated in culture medium M16/mix AA for $4 \mathrm{~h}$. (a) Mean absolute amino acid depletion rates (pmol per embryo $h^{-1} \pm$ SEM). (b) Mean proportional amino acid depletion rates (\% per embryo $h^{-1} \pm$ SEM) ${ }^{*} P<0.05,{ }^{* *} P<0.01,{ }^{* * *} P<0.001$ compared with zero depletion.

\section{Embryo volume}

On day 5, embryos grown in M16/mix AA had a significantly greater volume than those grown in the absence of amino acids $(P<0.001)$ (Fig. 6). The same effect was observed on day 6 although, while significant, the difference was not so great $(P<0.05)$.

All groups of embryos appeared morphologically normal throughout the experimental period.

\section{Discussion}

This is the first report of the consumption of a mixture of amino acids by preimplantation mouse embryos at the blastocyst stage. Mouse blastocysts are normally found in the uterus, but there are no reports on amino acid concentrations in mouse uterine fluid. Two strategies were therefore adopted. The first was to use an amino acid mixture in which the concentrations

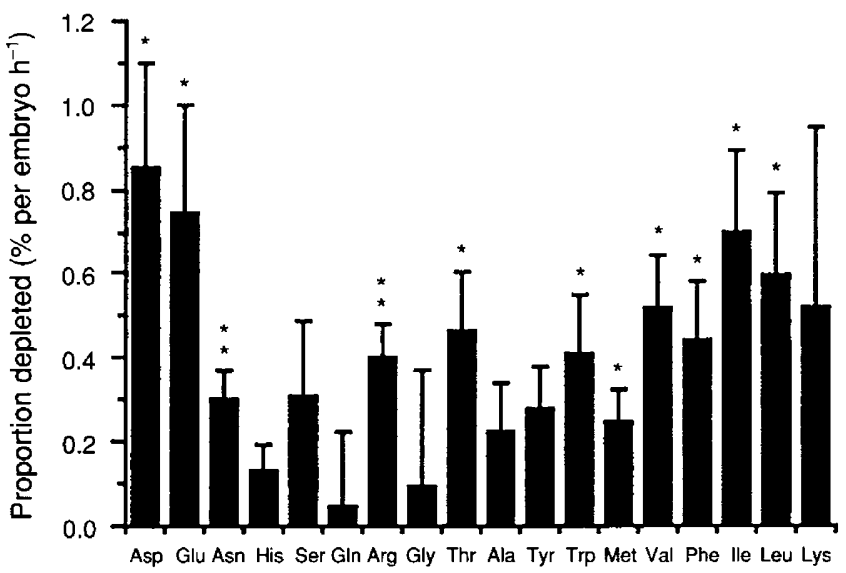

Amino acid

Fig. 5. Mean proportional amino acid depletion rates (pmol per embryo $h^{-1} \pm$ SEM) by mouse embryos on day 5 incubated in culture medium M16/0.1 AA for $4 \mathrm{~h} .{ }^{*} P<0.05,{ }^{* *} P<0.01$ compared with zero depletion.

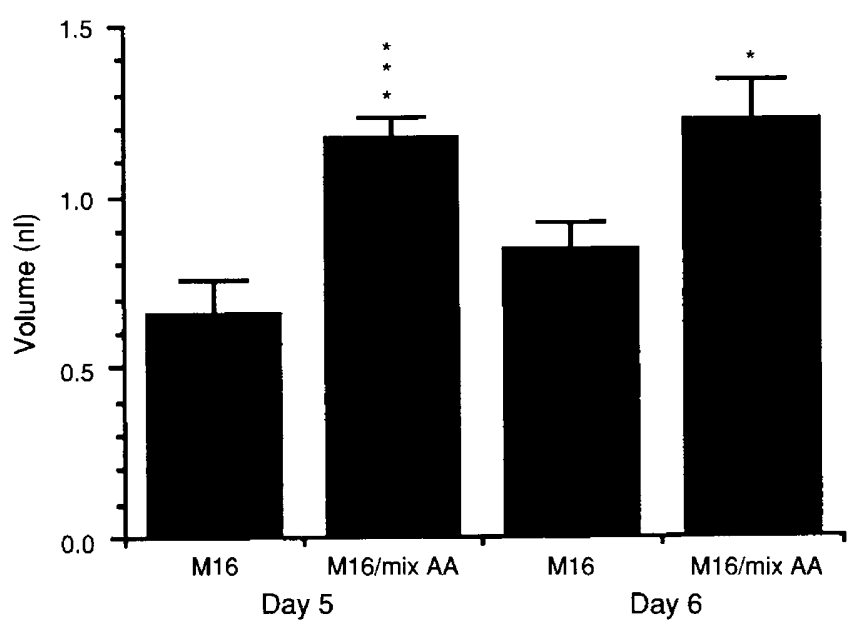

Fig. 6. Mean blastocyst volume $(\mathrm{nl} \pm$ SEM) of mouse embryos grown in MI6 and M16/mix AA media. Embryos were flushed from the uterus on day 4 after hCG treatment and grown in culture until day 6 . ${ }^{*} P<0.05,{ }^{* * *} P<0.001$ compared with M16 on the same day.

of essential amino acids were similar to those shown to support optimal growth of peri-implantation embryos in vitro (Spindle and Pedersen, 1973); the concentrations of the nonessential amino acids were based on the data of Miller and Schultz (1987) for rabbit oviduct and uterine fluids and on medium 199. The second strategy was to incubate embryos with a mixture of amino acids each at $0.1 \mathrm{mmol} \mathrm{l}^{-1}$. Protein was not included in the culture medium to avoid the problems of possible degradation and the introduction of protein-bound contaminants, which could have obscured the use of the amino acid mixtures by embryos.

HPLC, following derivatization with $o$-phthaldialdehyde, has been used to measure the concentrations of individual amino acids in the medium after incubation, enabling the depletion of a given amino acid to be measured in the presence of others.

Blastocysts were chosen for the study, since substantial growth of the preimplantation embryo occurs at this stage 
when amino acids are included in the culture medium (Lamb and Leese, 1993). The volumes of embryos grown between days 4 and 5 and between days 5 and 6 in M16/mix AA were significantly greater than those cultured in M16 alone. The fact that the presence of amino acids enhanced blastocyst expansion suggests that the concentrations used can sustain embryo development.

It should be emphasized that the data do not represent a rigorous kinetic analysis of amino acid transport systems but rather a description of the preferences of embryos for amino acids when provided with a mixture. For uptake to occur there must be appropriate systems to transport amino acids into the embryo, where they are accumulated or metabolized. It is possible that a few amino acids may subsequently be secreted intact, or after interconversion. For example, the uptake of glutamate could be followed by its conversion to glutamine, which could then be exported from the embryo and obscure the uptake of glutamine from the medium. However, this seems unlikely.

Relatively few amino acids were depleted at significant rates, especially on day 4. More amino acids exhibit significant depletion rates, and the average rate of depletion is higher on day 5 than on day 4 .

The proportional depletion rate demonstrates which amino acids are being depleted by embryos when the initial amino acid concentration is taken into account. There were about twice as many amino acids depleted at significant rates on day 5 than on day 4 , indicating a need for a wider range of amino acids at this later developmental stage. On day 5 , there appears to be a greater requirement for the aromatic amino acids, along with asparagine, threonine, methionine and valine, than on day 4. Aspartate, glutamate, arginine, isoleucine and leucine are the amino acids that exhibited significant depletion on both days tested. It is notable that aspartate and glutamate are involved in the metabolic pathways of transamination and deamination, which allow amino acids to be interconverted or degraded. Such a role is consistent with the marked increase in metabolism that accompanies blastocyst formation in mice (Leese, 1991). Of the other amino acids, the most interesting is leucine, which is important in the control of protein turnover and is the essential amino acid required in largest amounts by infants under 6 months of age (Newsholme and Leech, 1983).

Previous reported uptake rates for amino acids tend to be considerably higher than those found in this study (Van Winkle, 1988; Lewis and Kaye, 1992). This may be because the present method detects only amino acid concentrations in the medium, and gives no indication of movement into and out of the embryo. However, apart from this study, amino acids have been added to media singly or in pairs, often at high concentrations; neither situation is one that embryos would normally encounter. The depletion rates reported in this study are likely to be more representative of the requirements of the embryo in vivo, since competition between amino acids can occur throughout the incubation period. However, this makes comparisons with other reports difficult, as amino acid movement into the embryo over several hours, with subsequent metabolism or incorporation into protein, is a complex process that cannot easily be ascribed to specific transport systems.

The rates of depletion of amino acids did not appear to be related to sizes of the amino acid pool of mouse blastocysts reported by Schultz et al. (1981). Such intracellular or blastocoel cavity pools will be a complex function of a number of components including amino acid uptake and metabolism by the trophectoderm, exit of amino acids into the cavity, and uptake and metabolism by the inner cell mass. It is therefore not surprising that there is no simple relationship between medium depletion rates and the content of amino acids in the blastocyst.

The embryos did not appear to distinguish between essential and nonessential amino acids; both categories have been shown to enhance blastocyst hatching in different studies (Spindle and Pedersen, 1973; Gardner and Lane, 1993).

Glutamine was not depleted at significant rates in any of the four situations tested, while the other three acidic or amide amino acids (aspartate, glutamate and asparagine) showed considerable depletion, especially on day 5 . There is considerable interest in the effect of glutamine on the development of preimplantation mammalian embryos. It is a constituent of the medium CZB (Chatot ef al., 1989) that enables embryos from random-bred mice to progress through the 'two-cell block', and of other related media. Its uptake has been measured in a number of studies (Gardner et al., 1989; Chatot et al., 1990; Lewis and Kaye, 1992), in which rates of $0.8-2.0$ pmol per embryo $\mathrm{h}^{-1}$ have been reported at the blastocyst stage.

Glycine, which has been proposed to act as an organic osmolyte (Van Winkle et al., 1990b), only exhibited significant depletion from M16/mix AA on day 4. This would not, however, preclude its accumulation to a high concentration in the blastocoel cavity, as reported by Van Winkle (1990b), since such accumulation is a complex function of the various transport and metabolic processes present in blastocysts, as indicated above.

Our investigation illustrates some of the complex processes that occur when embryos are exposed to a mixture of amino acids. A future approach to unravel the complexity of amino acid uptake further could be the administration of individually labelled amino acids in the presence of a physiological mixture.

The authors thank A. E. Douglas for assistance with the amino acid analysis and T. J. Crawford, J. G. Thompson and R. G. Wales for assistance with statistics. V. K. Lamb is in receipt of a SERC Studentship.

\section{References}

Brinster RL (1971) Uptake and incorporation of amino acids by the preimplantation mouse embryo Joumal of Reproduction and Fertility 27 329-338

Carney EW and Bavister BD (1987) Stimulatory and inhibitory effects of amino acids on the development of hamster eight-cell embryos in vitro loumal of In Vitro Fertilization and Embryo Transfer 4 162-167

Chat tot CL, Ziomek CA, Bavister BD, Lewis JL and Torres I (1989) An improved culture medium supports development of random-bred 1-cell mouse embryos in vitro Joumal of Reproduction and Fertility $86679-688$

Chat ot CL, Tasca RJ and Ziomek CA (1990) Glutamine uptake and utilization by preimplantation mouse embryos in $\mathrm{CZB}$ medium Journal of Reproduction and Fertility 89 335-346

Gardner DK and Lane $\mathbf{M}$ (1993) Amino acids and ammonium regulate mouse embryo development in culture Biology of Reproduction 48 377-385

Gardner DK, Clarke RN, Lechene CP and Biggers JD (1989) Development of a noninvasive ultramicrofluorometric method for measuring net uptake of glutamine by single preimplantation mouse embryos Gamete Research $\mathbf{2 4}$ $427-438$ 
Hobbs JG and Kaye PL (1985) Glycine transport in mouse eggs and preimplantation embryos Joumal of Reproduction and Fertility 74 77-86

Jones BN, Pääbo S and Stein S (1981) Amino acid analysis and enzymatic sequence determination of peptides by an improved o-phthaldialdehyde precolumn labeling procedure Journal of Liquid Chromatography 4 565-586

Kaye PL (1986) Metabolic aspects of the physiology of the preimplantation embryo. In Experimental Approaches to Mammalian Embryonic Development pP 267-292 Eds J Rossant and RA Pedersen. Cambridge University Press, New York

Lamb VK and Leese HJ (1993) Factors involved in the onset of growth in early mouse embryos Joumal of Reproduction and Fertility Abstract Series 11 Abstract 129

Leese HJ (1991) Metabolism of the preimplantation mammalian embryo Oxford Reviews of Reproductive Biology 13 35-72

Leese HJ, Aldridge S and Jeffries KS (1979) The movement of amino acids into rabbit oviductal fluid Joumal of Reproduction and Fertility 56 623-626

Lewis AMcD and Kaye PL (1992) Characterization of glutamine uptake in mouse two-cell embryos and blastocysts Journal of Reproduction and Fertility $95221-229$

Miller JGO and Schultz GA (1987) Amino acid content of preimplantation rabbit embryos and fluids of the reproductive tract Biology of Reproduction 36 125-129

Newsholme EA and Leech AR (1983) Biochemistry for the Medical Sciences. John Wiley and Sons, New York
Quinn P, Barros C and Whittingham DG (1982) Preservation of hamster oocytes to assay the capacity of human spermatozoa Journal of Reproduction and Fertility 66 161-168

Schultz GA, Kaye PL, McKay DJ and Johnson MH (1981) Endogenous amino acid pool sizes in mouse eggs and preimplantation embryos Joumal of Reproduction and Fertility 61 387-393

Spindle AI and Pedersen RA (1973) Hatching, attachment, and outgrowth of mouse blastocysts in vitro: fixed nitrogen requirements Journal of Experimental Zoology 186 305-318

Van Wirkle LJ (1988) Amino acid transport in developing animal oocytes and early conceptuses Biochimica et Biophysica Acta 947 173-208

Van Winkle LJ, Campione AL, Gorman JM and Weimer BD (1990a) Changes in the activities of amino acid transport systems $\mathrm{b}^{\mathrm{O}+}$ and $\mathrm{L}$ during development of preimplantation mouse conceptuses Biochimica et Biophysica Acta $102177-84$

Van Winkle LJ, Haghighat $\mathbf{N}$ and Campione AL (1990b) Glycine protects preimplantation mouse conceptuses from a detrimental effect on development of the inorganic ions in oviductal fluid Journal of Experimental Zoology $253215-219$

Van Winkle LJ, Mann DF, Weimer BD and Campione AL (1991) $\mathrm{Na}^{+}$-dependent transport of anionic amino acids by preimplantation mouse blastocysts Biochimica et Biophysica Acta 1068 231-236

Whittingham DG (1971) Culture of mouse ova Joumal of Reproduction and Fertility Supplement 14 7-21 